\title{
BMJ Open Efficacy of left ventricular unloading strategies during venoarterial extracorporeal membrane oxygenation in patients with cardiogenic shock: a protocol for a systematic review and Bayesian network meta-analysis
}

Pengbin Zhang, ${ }^{1,2}$ Shilin Wei, ${ }^{1,3}$ Kerong Zhai, ${ }^{1,3}$ Jian Huang, ${ }^{1,3}$ Xingdong Cheng, ${ }^{1,3}$ Zhenze Tao, ${ }^{1,3}$ Bingren Gao (D) , ${ }^{1}$ Debin Liu (D) , ${ }^{1}$ Yongnan Li (D) ${ }^{1,3}$

To cite: Zhang P, Wei S, Zhai K, et al. Efficacy of left ventricular unloading strategies during venoarterial extracorporeal membrane oxygenation in patients with cardiogenic shock: a protocol for a systematic review and Bayesian network meta-analysis. BMJ Open 2021;11:e047046. doi:10.1136/ bmjopen-2020-047046

- Prepublication history and additional supplemental material for this paper are available online. To view these files, please visit the journal online (http://dx.doi.org/10.1136/ bmjopen-2020-047046)

PZ and SW contributed equally.

Received 17 November 2020 Accepted 27 September 2021

Check for updates

(c) Author(s) (or their employer(s)) 2021. Re-use permitted under CC BY-NC. No commercial re-use. See rights and permissions. Published by BMJ.

For numbered affiliations see end of article.

Correspondence to Professor Debin Liu; ery_liudb@|zu.edu.cn and Professor Yongnan Li; lyngyq2006@foxmail.com

\section{ABSTRACT}

Introduction Venoarterial extracorporeal membrane oxygenation (VA-ECMO) has been widely used for patients with refractory cardiogenic shock. A common side effect of this technic is the resultant increase in left ventricular (LV) afterload which could potentially aggravate myocardial ischaemia, delay ventricular recovery and increase the risk of pulmonary congestion. Several LV unloading strategies have been proposed and implemented to mitigate these complications. However, it is still indistinct that which one is the best choice for clinical application. This Bayesian network meta-analysis (NMA) aims to compare the efficacy of different LV unloading strategies during VA-ECMO. Methods and analysis PubMed, Embase, the Cochrane Library and the International Clinical Trials Registry Platform will be explored from their inception to 31 December 2020. Random controlled trials and cohort studies that compared different LV unloading strategies during VA-ECMO will be included in this study. The primary outcome will be in-hospital mortality. The secondary outcomes will include neurological complications, haemolysis, bleeding, limb ischaemia, renal failure, gastrointestinal complications, sepsis, duration of mechanical ventilation, length of intensive care unit and hospital stays. Pairwise and NMA will respectively be conducted using Stata (V.16, StataCorp) and Aggregate Data Drug Information System (V.1.16.5), and the cumulative probability will be used to rank the included LV unloading strategies. The risk of bias will be conducted using the Cochrane Collaboration's tool or Newcastle-0ttawa Quality Assessment Scale according to their study design. Subgroup analysis, sensitivity analysis and publication bias assessment will be performed. The Grading of Recommendations Assessment, Development and Evaluation will be conducted to explore the quality of evidence.

Ethics and dissemination Either ethics approval or patient consent is not necessary, because this study will be based on literature. The results will be disseminated through peer-reviewed publications and conference presentations.
Strengths and limitations of this study

- This network meta-analysis (NMA) will address the problem that which strategy could achieve left ventricular (LV) unloading most effectively during venoarterial extracorporeal membrane oxygenation (VA-ECMO) and increase cardiogenic shock patient survival benefit, and will provide evidence for clinical decision making.

- This NMA will combine all direct with indirect evidence within a Bayesian framework by Markov Chain Monte Carlo simulation, and it is anticipated to be the first Bayesian NMA that evaluates the efficacy of different LV unloading strategies during VA-ECMO.

- The quality of evidence will be assessed by the Grade of Recommendations Assessment, Development and Evaluation system, and statistical inconsistency assessment, subgroup analysis, sensitivity analysis and publication bias assessment will be performed in this NMA

- At present, there has no standardised diagnostic criteria for LV overload or no guidelines for the timing of intervention for it, and there may be significant heterogeneity and inconsistency in this NMA.

PROSPERO registration number CRD42020165093.

\section{INTRODUCTION}

Cardiogenic shock (CS) remains a challenging condition with about 40\%-50\% mortality, in spite of significant advances have been achieved in revascularisation and heart failure pharmacotherapies. ${ }^{1-4}$ Currently, venoarterial extracorporeal membrane oxygenation (VA-ECMO) has been widely used to provide life support for these refractory CS patients. ${ }^{5}{ }^{6}$ VA-ECMO could be used as a bridge to myocardial recovery, durable 
mechanical circulatory support or heart transplant for these patients. ${ }^{7}$ Recently, some observational studies found that VA-ECMO was associated with a significant mortality benefit and had significantly improved outcomes of patients. ${ }^{8-10}$ What but is worth reminding, the results are need to be proved by large randomised trials like the ongoing extracorporeal life support trials.

Despite the benefits of VA-ECMO, it also has potentially deleterious effect like the increased left ventricular (LV) afterload. ${ }^{11}$ VA-ECMO works relying on generating varying degrees of retrograde aortic flow, that will increase afterload in the aorta. The increased LV afterload could cause an increase in wall stress and oxygen demand, exacerbate myocardial ischaemia, and delaying recovery from CS. ${ }^{12}{ }^{13}$ Elevated LV pressure could further promote LV dilatation, trigger ventricular arrhythmias, and result in elevated left atrial (LA) pressure causing pulmonary oedema. ${ }^{14}$ Eventually, the reduced flow across the aortic valve and retrograde aortic flow can induce the LV or aortic root thrombus. ${ }^{15}$ Therefore, the increased $\mathrm{LV}$ afterload was considered as a limitation to the success of VA-ECMO in the presence of CS and associated with high rates of morbidity and mortality. ${ }^{16}$

Several interventions have been used to unload LV afterload, thereby reduce the complications associated with increased LV afterload. ${ }^{17}$ Recent meta-analyses based on retrospective observational studies had demonstrated that VA-ECMO concomitant with LV unloading was associated with higher short-term survival benefit as compared with VA-ECMO alone. ${ }^{18-20}$ According to intraaortic balloon pump (IABP)-SHOCK II trial, no reduction in mortality was found in patients with myocardial infarction complicated by CS adjunctive IABP. ${ }^{21}$ However, despite conflicting results, IABP was usually used concomitantly with VA-ECMO to unload the LV afterload and accelerate LV emptying in clinical practice. In addition, our previous study demonstrated VA-ECMO plus IABP was associated with decreased mortality. ${ }^{22}$

Nevertheless, lacking direct randomised trials or studies comparing one with all other LV unloading strategies, it is still controversial that which strategy could achieve LV unloading and increase survival benefit most effectively for CS patients. Network meta-analysis (NMA) could combine all available evidence and compare the effectiveness of all available LV unloading strategies. ${ }^{23-25}$ Therefore, we aim to conduct the Bayesian NMA to address the thorny clinical problem: which strategy could achieve LV unloading and increase the survival benefit most effectively. Besides, in light of lacking randomised evidence supporting a survival benefit for VA-ECMO, this meta-analysis will add to the present knowledge of how to improve clinical outcome of CS patients supported by VA-ECMO.

\section{Objective}

The objective of this study is to synthesise the available evidence on LV unloading strategies during VA-ECMO and compare the efficacy of different LV unloading strategies for CS patients using a Bayesian NMA.

\section{METHODS AND ANALYSIS \\ Overview}

This protocol is written following the Preferred Reporting Items for Systematic Reviews and Meta-Analyses Protocols (PRISMA-P) guidelines ${ }^{26}$ and the results of this NMA will be reported according to the PRISMA statement and PRISMA extension for NMA (PRISMA-NMA). ${ }^{24} 27$ The PRISMA-P checklist for this study is included in online supplemental file 1. The Bayesian framework by Markov Chain Monte Carlo simulation will be used in this study.

\section{Patient and public involvement}

No patients or public involvement will be involved in this study.

\section{Information source and search strategy}

PubMed, Embase, the Cochrane Library and the International Clinical Trials Registry Platform will be explored from their inception to 31 December 2020. We will use a combination of MeSH, EMTREE and free-text terms: 'extracorporeal membrane oxygenation', 'extracorporeal life support', 'intra-aortic balloon pumping', 'counterpulsation', 'impella', 'TandemHeart' 'transaortic catheter', 'transseptal left atrial cannula', 'decompression', 'venting', 'unloading'. The search strategy will be implemented by two experienced scholars of information retrieval (DL and YL). Any potentially relevant article will be retrieved for review. Besides, references of included studies and narrative reviews and meta-analyses will be considered for additional potential studies. There will be no restrictions on date limit, country, the language of publication, publication status or year of publication. The search strategies are shown in online supplemental file 2.

\section{Eligibility criteria}

Type of study

All published clinical studies investigating VA-ECMO and reporting data on LV unloading strategies will be evaluated for inclusion in this meta-analysis. Random controlled trials and prospective or retrospective cohort studies, reporting at least 10 adult CS patients, that compare different LV unloading technique during VA-ECMO will be included in this study. Case-control and cross-sectional studies, case series studies, reviews and meta-analyses, letters to the editor, case reports, expert opinions and animal studies will be excluded.

\section{Types of participants}

Adult patients (older than 18 years) with CS using VA-ECMO with or without LV unloading will be included. We will not apply restrictions about gender, ethnic origin or other characteristics.

Types of interventions and comparators

We will include any LV unloading strategies adjunct to VA-ECMO covering surgical LV unloading strategy, IABP, 


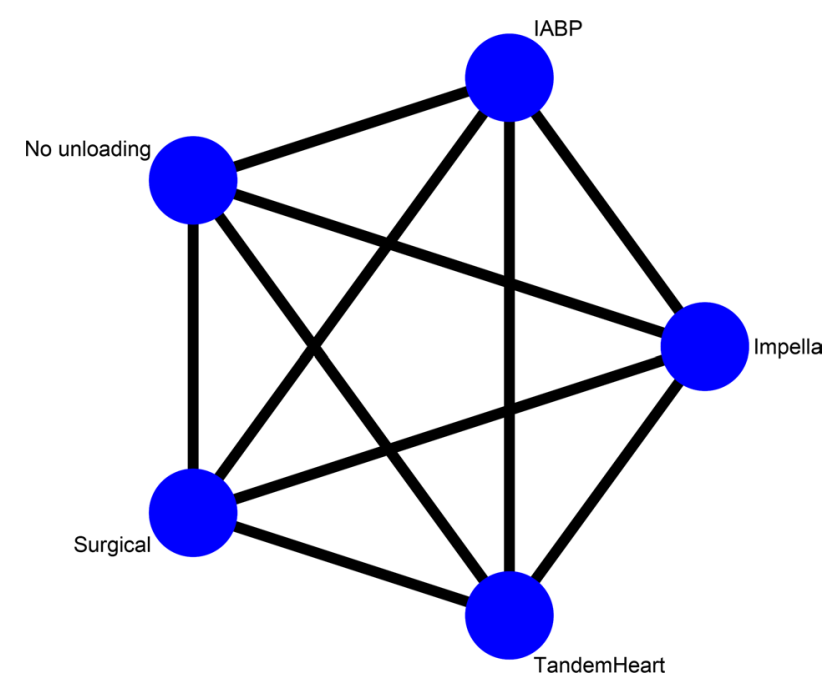

Figure 1 The network of all possible pairwise comparisons among the eligible interventions. IABP, intra-aortic balloon pump.

Impella and TandemHeart. ${ }^{12} 28-30$ Despite the common comparator is VA-ECMO alone, any LV unloading technique adjunct to VA-ECMO could be intervention or comparator in pairwise and NMA. The network of all possible pairwise comparisons among the eligible interventions is shown in figure 1 . As the surgical techniques of $L V$ venting could be achieved by many approaches, we define the surgical LV unloading strategy as follows: (1) implanting LV venting cannulation of the ventricle apex or through the mitral valve from the LA; (2) implanting a catheter across the aortic valve percutaneously; (3) implanting LV venting surgical cannulation through right superior pulmonary vein, LA roof or interatrial groove into LA; (4) transseptal LA cannula; (5) an interatrial septostomy (septostomy usually with ballooning or stent); (6) the surgical or percutaneous pulmonary artery cannulation and (7) simultaneous left and right atrial drainage with the multistage cannula coming from the femoral vein and positioned transeptally. ${ }^{12} 182831$

\section{Type of outcomes}

Primary outcome

The primary outcome was all-cause in-hospital mortality (death of ECMO withdrawal due to futility, of patients unable to be weaned off ECMO, and of patients who died before hospital discharge despite successful ECMO weaning).

\section{Secondary outcomes}

The secondary outcomes will be neurological complications, haemolysis, bleeding, limb ischaemia, renal failure, gastrointestinal complications, sepsis, duration of mechanical ventilation, length of intensive care unit and hospital stays.

\section{Study selection}

All studies to be screened will be managed by Endnote V.X9 (Thomson-Reuters; 2018, New York, USA). First, it will be used to classify and organise the preliminary literature and exclude repeated literature. Second, following the prespecified inclusion criteria, two independent reviewers (PZ and SW) will screen the title and abstract of each study independently and identify relevant studies. Third, they will obtain and review the full text of all potential studies, then, they will make decisions independently and compare their selection of studies. Any discrepancies will be resolved by consensus. If consensus cannot be reached, DL and YL will serve as arbiters. And if discrepancy is caused by insufficient information of the literature, it is necessary to classify it into the category of waiting for evaluating and then decide whether it should be included after adding sufficient additional information. If studies have duplicate data, only the study with larger sample size or longer follow-up time will be included. The proposed flow diagram of studies selection is illustrated in figure 2.

\section{Data extraction and management}

Microsoft Excel (V.2019; Microsoft, USA) will be used to extract data from the included studies by two reviewers ( $\mathrm{PZ}$ and SW) independently, using a standardised data extraction form. Missing data will be requested from study authors. Any discrepancies will be resolved by consensus. If consensus cannot be reached, DL and YL will serve as arbiters. The characteristics of the extracted data items are shown in table 1 .

\section{Risk of bias assessment}

Risk of bias will be assessed using the Cochrane risk of bias tool or Newcastle-Ottawa Quality Assessment Scale (NOS) for random controlled trials or observational studies separately ${ }^{32} 33$ The Cochrane Collaboration's risk of bias assessment tool includes the following seven domains: random sequence generation, allocation concealment, blinding of participants and personnel, blinding of outcome assessment, incomplete outcome data, selective reporting, and other bias. Each item will be classified into one of three categories as follows: unclear, high or low risk. The NOS will be used to assess each included observational study using 'star system'. Each study will be judged on three broad perspectives: the selection of the study groups, the comparability of the groups, and the ascertainment of outcome of interest. A total score of 5 or less is considered low, 6 or 7 is considered moderate, and 8 or 9 is deemed of high quality. The judgements will be made by two review authors (PZ and SW) independently. Any discrepancies will be resolved by consensus. If consensus cannot be reached, DL and YL will serve as arbiters.

\section{Data synthesis and analysis}

When quantitative analysis cannot be conducted, we will narratively describe the results. If the quantitative analysis is feasible, statistical analyses will be conducted using Stata (V.16, StataCorp) and (V.1.16.5 Aggregate Data Drug Information System, http://drugis.org/addis). The binary outcomes and continuous outcomes will be 


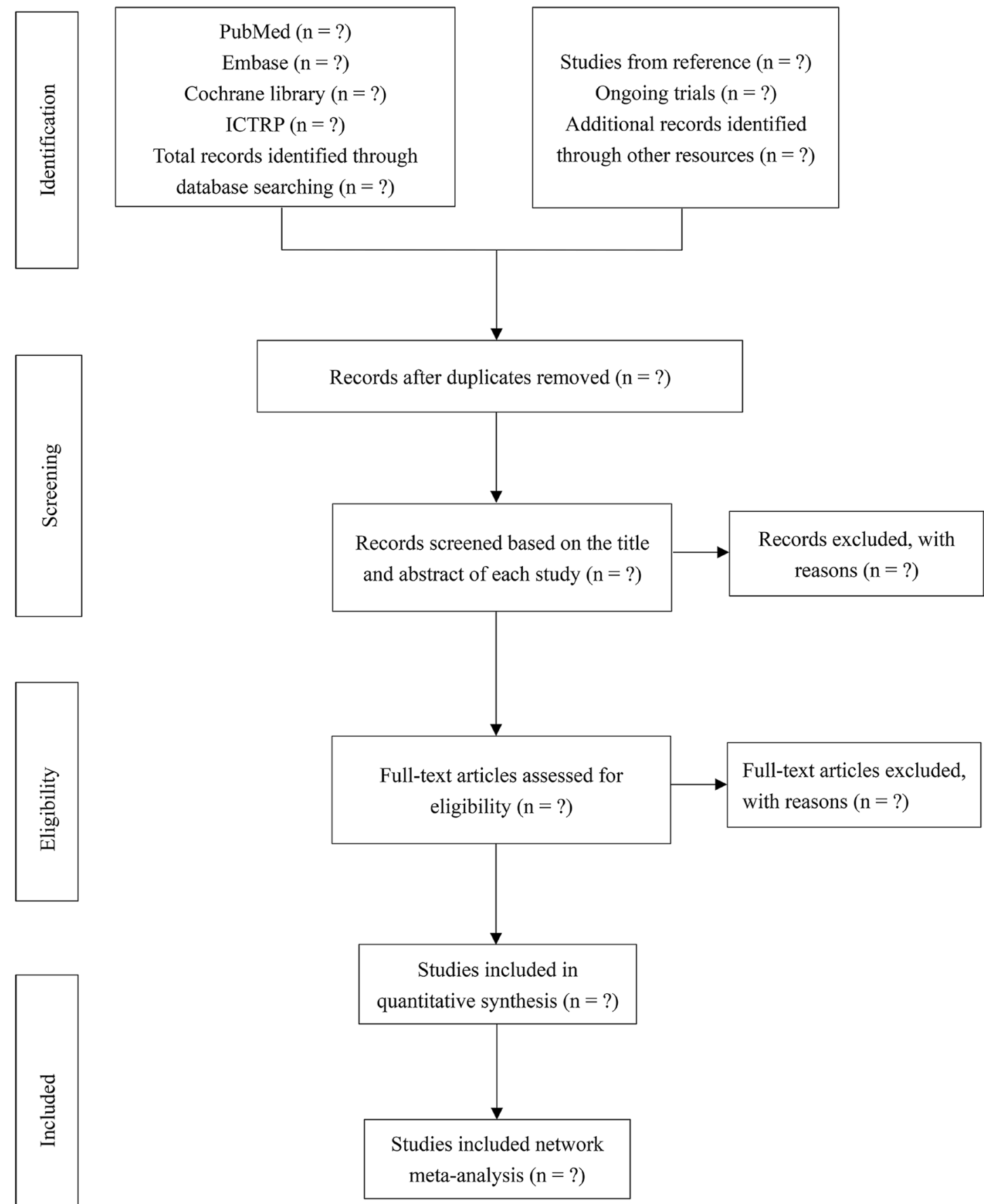

Figure 2 Hypothetic flow diagram of the study selection process. ICTRP, International Clinical Trials Registry Platform.

presented as risk ratios or mean difference with their $95 \%$ CIs, respectively.

\section{Pairwise meta-analyses}

All the direct comparisons will be performed using Stata software and random-effects model if no less than two studies. Methodological and clinical diversity always exist in pairwise meta-analysis, statistical heterogeneity is inevitable. ${ }^{34}$ Cochran's $Q$ tests, $I^{2}$ statistic, and visual inspection of forest plots will be used to assess heterogeneity levels. If significant heterogeneity exists $\left(\mathrm{I}^{2} \geq 50 \%\right.$ or $\left.\mathrm{p}<0.1\right)$, subgroup or sensitivity analysis or meta-regression will be used to explain the source of heterogeneity. Moreover, if there is considerable heterogeneity, especially when the direction of the effect is inconsistent, we will do a general statistical description.

\section{Indirect and mixed comparisons of interventions}

A random-effects NMA within the Bayesian framework will be applied. Interactions among all included studies will be shown in the network geometry, and the contribution plot for the network will show the contributions of direct comparisons. ${ }^{35}$ In the network diagram, the dots will represent every intervention, and the size of the dot will mean the number of participants. The lines will indicate direct comparisons between different interventions 
Table 1 Characteristics of the extracted data items

\begin{tabular}{ll}
\hline Characteristics & Data items \\
\hline Study characteristics & $\begin{array}{l}\text { Title, first author, journal, publication year, study period, study type, country/district, ELSO centre, } \\
\text { funding information. }\end{array}$ \\
Participants & $\begin{array}{l}\text { Sample size, gender, age, underlying diseases, duration of disease, LV ejection fraction, aetiology of } \\
\text { cardiogenic shock, no of peripheral ECMO, average time on ECMO, no of survive to D/C, no of bridged } \\
\text { to VAD/no of survive to D/C, no of bridged to HTP/no of survive to D/C, ECPR. }\end{array}$ \\
Interventions & $\begin{array}{l}\text { No of patients with LV unloading, the diagnose of LV overload, strategy of LV unloading, the time of LV } \\
\text { unloading. }\end{array}$ \\
Comparisons & $\begin{array}{l}\text { No of patients without LV unloading. } \\
\text { Outcomes }\end{array}$ \\
$\begin{array}{l}\text { Primary outcome: all-cause in-hospital mortality. } \\
\text { Secondary outcomes: neurological complications, haemolysis, bleeding, limb ischaemia, renal failure, } \\
\text { gastrointestinal complications, sepsis, duration of mechanical ventilation, length of intensive care unit } \\
\text { and hospital stays. }\end{array}$
\end{tabular}

D/C, hospital discharge; ECMO, extracorporeal membrane oxygenation; ECPR, extracorporeal cardiopulmonary resuscitation; ELSO,

Extracorporeal Life Support Organisation; HTP, heart transplant; LV, left ventricular; VAD, ventricular assist device.

and the thickness of the line will mean the amount of studies ${ }^{36}$ For each outcome, we will present the contribution plots, which exposit the contribution of each direct comparison to the entire network as well as for each network estimate. ${ }^{37}$ The main characteristics of NMA are ranking analysis having the ability to rank the various treatments for each outcome. The cumulative probability will be used to rank the included LV unloading strategies.

\section{Assessment of inconsistency}

Inconsistency is the statistical manifestation of the violation of the transitivity assumption. It is the differences between indirect and direct effect estimates for the same comparison, includes loop inconsistency and design inconsistency. ${ }^{38}$ We will use the node-splitting method to evaluate the inconsistency between direct and indirect evidence locally. If $\mathrm{p}>0.05$, it suggests consistency between direct and indirect evidence. We will also investigate possible sources of inconsistency using inconsistency factor (IF) among studies in each closed loop. If the 95\% CIs of IF values include zero, it indicates that there is no significant inconsistency.

\section{Subgroup analysis and sensitivity analysis}

If there are sufficient data, we will conduct prespecified subgroup analyses for outcomes based on following: (1) aetiology of CS: postcardiotomy shock; acute myocardial infarction; myocarditis; mixed etiologies; (2) quality of study; (3) the time of LV unloading; (4) the mechanism of LV decompression. Besides, the clinical data on the haemodynamic changes induced by LV unloading will be added into analysis. If available, the 24-hour haemodynamic changes (including the changes in LA pressure, pulmonary capillary wedge pressure (PCWP), pulmonary systolic pressure (PSP), pulmonary diastolic pressure (PDP), central venous pressure (CVP), LV end-diastolic pressure and mixed venous oxygen saturation) after implementing any LV unloading strategies will be extracted from the studies into the analysis. To correlate our findings with changes in haemodynamics, a metaregression between the 24-hour haemodynamic changes (including the changes in LA pressure, PCWP, PSP, PDP, CVP, LV end-diastolic pressure and mixed venous oxygen saturation if available) induced by any LV unloading strategies and primary or secondary outcomes will be implemented. Moreover, if available, the haemodynamic condition of the $\mathrm{LV}$ will be illustrated by the LV pressure-volume (PV) loop, and we will summarise all PV loop (during CS and following LV unloading) into one figure. Meanwhile, the sensitivity analysis will also be conducted to validate the robustness of the results by excluding each study.

\section{Assessment of publication bias}

To assess small study effects and publication bias, funnel plot will be used in pairwise meta-analyses when at least 10 studies would be analysed. The comparison-adjusted funnel plot will be employed to identify possible small-study effects including publication bias at the network level. ${ }^{37}$ And Egger's test will be used to assess the symmetry of the funnel plot. ${ }^{39}$

\section{Quality of evidence}

The Grading of Recommendations Assessment, Development and Evaluation (GRADE) guidelines will be used to assess the quality of direct and indirect evidence for the main outcomes. ${ }^{40}$ Five factors can reduce the quality of evidence: study limitations (risk of bias), inconsistency, indirectness, publication bias and imprecision. Correspondingly, three factors can improve the quality of evidence: residual confounding, dose-response gradient, and large magnitude of effect. The quality of evidence will be graded in four levels: very low, low, moderate, high. 


\section{DISCUSSION}

VA-ECMO has become the preferred device for shortterm haemodynamic support in CS patients as cost, ease and rapidity of cannulation, and the ability to provide biventricular and respiratory support. ${ }^{5} 641$ Despite the improvements in technology have mitigated many adverse effects like the interaction between artificial surfaces of ECMO circuits and blood, ${ }^{42}$ the issue that a resultant increase in $\mathrm{LV}$ afterload during VA-ECMO has not been solved. Several interventions have been used to achieve LV unloading, however, it remains controversial that which unloading strategy could achieve LV unloading and increase survival benefit most effectively for CS patients. This Bayesian NMA will provide evidence for efficacy of different LV unloading strategies during VA-ECMO. As far as we know, this NMA is anticipated to be the first Bayesian NMA that assesses different LV unloading strategies during VA-ECMO. We expect that our findings will provide the best available evidence on LV unloading during VA-ECMO for clinicians, patients, cardiologists and practice guideline developers. And this NMA will help both clinical practice and study design in the future. This NMA may have some limitations. First, at present, the confusing problem still is the lack of evidence from large randomised trials that VA-ECMO could provide a significant survival benefit for CS patients. This may lead to some experts are sceptical about VA-ECMO for the management of CS. Second, although LV overload has been reported in many studies, there are still no standardised diagnostic criteria for LV overload and no guidelines for the timing of intervention for it. ${ }^{128}$ We will not limit the time for LV unloading, so the time of the intervention of LV overload in different clinical studies may be not unified absolutely. Besides, RCTs and cohort studies will be included in our study. All of the above will generate potential heterogeneity which may influence the results. Based on the characteristic of NMA that it is a statistical combination of all available evidence for an outcome from several studies, there may exist inconsistency in this NMA. And the inconsistency will be reduced by setting subgroups or conducting metaregression. However, it is also worth mentioning that if the scope of included studies is small, the ability to explore heterogeneity and conduct meta-regression could be limited.

\section{ETHICS AND DISSEMINATION}

This work synthesises evidence from previously published studies, there are no ethical concerns nor informed consent required, confirmed by the medical ethics committee of Lanzhou University Second Hospital. In addition, the data about this study will be submitted to the research management centre of Lanzhou University Second Hospital and kept for 5 years. This study is expected to provide an efficacy ranking of various $\mathrm{LV}$ unloading strategies during VA-ECMO in CS patients. Based on this NMA, clinicians and perfusionists will make a more accurate and optimal intervention for LV overload for adults with VA-ECMO. We will publish the findings and results of this study in a peer-reviewed journal.

\section{Author affiliations}

${ }^{1}$ Department of Cardiac Surgery, Lanzhou University Second Hospital, Lanzhou, Gansu, China

${ }^{2}$ State Key Laboratory of Cardiovascular Disease, Fuwai Hospital, National Center for Cardiovascular Diseases, Chinese Academy of Medical Sciences and Peking Union Medical College, Beijing, China

${ }^{3}$ Laboratory of Extracorporeal Life Support, Lanzhou University Second Hospital, Lanzhou, Gansu, China

Contributors $\mathrm{PZ}, \mathrm{BG}, \mathrm{DL}$ and $\mathrm{YL}$ participated in the conception and design of the study. PZ, SW, KZ and YL were responsible for search strategy development. XC, ZT and SW tested the feasibility of this protocol. PZ, KZ and JH wrote the original draft. PZ, DL and YL reviewed the manuscript and approved the final manuscript as submitted. All authors contributed to the development of the selection criteria. All authors read, provided feedback and approved the final manuscript as submitted.

Funding This study was supported by the Cuiying Scientific and Technological Innovation Program of Lanzhou University Second Hospital (No. CY2019-QN01, No. CY2017-BJ01 and No. CY2017- MS07) and Innovation Fund Project of Higher Education in Gansu Province (2020B-028).

Competing interests None declared.

Patient consent for publication Not applicable.

Provenance and peer review Not commissioned; externally peer reviewed.

Supplemental material This content has been supplied by the author(s). It has not been vetted by BMJ Publishing Group Limited (BMJ) and may not have been peer-reviewed. Any opinions or recommendations discussed are solely those of the author(s) and are not endorsed by BMJ. BMJ disclaims all liability and responsibility arising from any reliance placed on the content. Where the content includes any translated material, BMJ does not warrant the accuracy and reliability of the translations (including but not limited to local regulations, clinical guidelines, terminology, drug names and drug dosages), and is not responsible for any error and/or omissions arising from translation and adaptation or otherwise.

Open access This is an open access article distributed in accordance with the Creative Commons Attribution Non Commercial (CC BY-NC 4.0) license, which permits others to distribute, remix, adapt, build upon this work non-commercially, and license their derivative works on different terms, provided the original work is properly cited, appropriate credit is given, any changes made indicated, and the use is non-commercial. See: http://creativecommons.org/licenses/by-nc/4.0/.

\section{ORCID iDs}

Bingren Gao http://orcid.org/0000-0002-2256-6203

Debin Liu http://orcid.org/0000-0002-1525-7509

Yongnan Li http://orcid.org/0000-0003-0821-8875

\section{REFERENCES}

1 Iborra-Egea O, Rueda F, García-García C, et al. Molecular signature of cardiogenic shock. Eur Heart J 2020;41:3839-48.

2 Thiele H, Ohman EM, de Waha-Thiele S, et al. Management of cardiogenic shock complicating myocardial infarction: an update 2019. Eur Heart J 2019;40:2671-83.

3 Thiele H, Ohman EM, Desch S, et al. Management of cardiogenic shock. Eur Heart J 2015;36:1223-30.

4 Reynolds HR, Hochman JS. Cardiogenic shock: current concepts and improving outcomes. Circulation 2008;117:686-97.

5 Werdan K, Gielen S, Ebelt H, et al. Mechanical circulatory support in cardiogenic shock. Eur Heart J 2014;35:156-67.

6 Guglin M, Zucker MJ, Bazan VM, et al. Venoarterial ECMO for adults: JACC scientific expert panel. J Am Coll Cardiol 2019;73:698-716.

7 Abrams D, Combes A, Brodie D. Extracorporeal membrane oxygenation in cardiopulmonary disease in adults. J Am Coll Cardiol 2014;63:2769-78.

8 Ouweneel DM, Schotborgh JV, Limpens J, et al. Extracorporeal life support during cardiac arrest and cardiogenic shock: a systematic review and meta-analysis. Intensive Care Med 2016;42:1922-34. 
9 Fordyce CB, Wang TY, Chen AY, et al. Long-term post-discharge risks in older survivors of myocardial infarction with and without outof-hospital cardiac arrest. J Am Coll Cardiol 2016;67:1981-90.

10 Chen S-W, Tsai F-C, Lin Y-S, et al. Long-term outcomes of extracorporeal membrane oxygenation support for postcardiotomy shock. J Thorac Cardiovasc Surg 2017;154:469-77.

11 Rupprecht L, Flörchinger B, Schopka S, et al. Cardiac decompression on extracorporeal life support: a review and discussion of the literature. Asaio J 2013;59:547-53.

12 Cevasco M, Takayama $\mathrm{H}$, Ando M, et al. Left ventricular distension and venting strategies for patients on venoarterial extracorporeal membrane oxygenation. J Thorac Dis 2019;11:1676-83.

13 Lorusso R. Are two crutches better than one? the ongoing dilemma on the effects and need for left ventricular unloading during veno-arterial extracorporeal membrane oxygenation. Eur J Heart Fail 2017;19:413-5.

14 Curran J, Burkhoff D, Kloner RA. Beyond reperfusion: acute ventricular unloading and cardioprotection during myocardial infarction. J Cardiovasc Transl Res 2019;12:95-106.

15 Hireche-Chikaoui H, Grübler MR, Bloch A, et al. Nonejecting hearts on femoral veno-arterial extracorporeal membrane oxygenation: aortic root blood stasis and thrombus formation-a case series and review of the literature. Crit Care Med 2018;46:e459-64.

16 Smith M, Vukomanovic A, Brodie D, et al. Duration of veno-arterial extracorporeal life support (Va ECMO) and outcome: an analysis of the extracorporeal life support organization (ELSO) registry. Crit Care 2017;21:45

17 Meani P, Gelsomino S, Natour E, et al. Modalities and effects of left ventricle unloading on extracorporeal life support: a review of the current literature. Eur J Heart Fail 2017;19 Suppl 2:84-91.

18 Kowalewski M, Malvindi PG, Zieliński K, et al. Left ventricle unloading with veno-arterial extracorporeal membrane oxygenation for cardiogenic shock. systematic review and meta-analysis. J Clin Med 2020;9:1039.

19 Russo JJ, Aleksova N, Pitcher I, et al. Left ventricular unloading during extracorporeal membrane oxygenation in patients with cardiogenic shock. J Am Coll Cardiol 2019;73:654-62.

20 Al-Fares AA, Randhawa VK, Englesakis M, et al. Optimal strategy and timing of left ventricular Venting during veno-arterial extracorporeal life support for adults in cardiogenic shock: a systematic review and meta-analysis. Circ Heart Fail 2019;12:e006486.

21 Thiele H, Zeymer U, Neumann F-J, et al. Intra-Aortic balloon counterpulsation in acute myocardial infarction complicated by cardiogenic shock (IABP-SHOCK II): final 12 month results of a randomised, open-label trial. Lancet 2013;382:1638-45.

22 Li Y, Yan S, Gao S, et al. Effect of an intra-aortic balloon pump with venoarterial extracorporeal membrane oxygenation on mortality of patients with cardiogenic shock: a systematic review and metaanalysist. Eur J Cardiothorac Surg 2019;55:395-404.

23 Riley RD, Jackson D, Salanti G, et al. Multivariate and network metaanalysis of multiple outcomes and multiple treatments: rationale, concepts, and examples. BMJ 2017;358:j3932.

24 Hutton B, Salanti G, Caldwell DM, et al. The PRISMA extension statement for reporting of systematic reviews incorporating network meta-analyses of health care interventions: checklist and explanations. Ann Intern Med 2015;162:777-84.

25 Mills EJ, Thorlund K, loannidis JPA. Demystifying trial networks and network meta-analysis. BMJ 2013;346:f2914.
26 Shamseer L, Moher D, Clarke M, et al. Preferred reporting items for systematic review and meta-analysis protocols (PRISMA-P) 2015: elaboration and explanation. BMJ 2015;350:g7647.

27 Liberati A, Altman DG, Tetzlaff J, et al. The PRISMA statement for reporting systematic reviews and meta-analyses of studies that evaluate health care interventions: explanation and elaboration. PLOS Med 2009;6:e1000100.

28 Xie A, Forrest P, Loforte A. Left ventricular decompression in venoarterial extracorporeal membrane oxygenation. Ann Cardiothorac Surg 2019;8:9-18.

29 Desai SR, Hwang NC. Strategies for left ventricular decompression during venoarterial extracorporeal membrane oxygenation - a narrative review. J Cardiothorac Vasc Anesth 2020;34:208-18.

30 Rao P, Khalpey Z, Smith R, et al. Venoarterial extracorporeal membrane oxygenation for cardiogenic shock and cardiac arrest. Circ Heart Fail 2018;11:e004905.

31 Li Y, Gao S, Cai L, et al. Left ventricle unloading strategy: which one is more effective in venoarterial extracorporeal membrane oxygenation patients? J Am Coll Cardiol 2019;73:3035-6.

32 Higgins JPT, Altman DG, Gøtzsche PC, et al. The Cochrane collaboration's tool for assessing risk of bias in randomised trials. BMJ 2011;343:d5928.

33 Stang A. Critical evaluation of the Newcastle-Ottawa scale for the assessment of the quality of nonrandomized studies in metaanalyses. Eur J Epidemiol 2010;25:603-5.

34 Higgins JPT, Thompson SG, Deeks JJ, et al. Measuring inconsistency in meta-analyses. BMJ 2003;327:557-60.

35 Salanti G, Ades AE, loannidis JPA. Graphical methods and numerical summaries for presenting results from multipletreatment meta-analysis: an overview and tutorial. $J$ Clin Epidemiol 2011;64:163-71.

36 Catalá-López F, Tobías A, Cameron C, et al. Network meta-analysis for comparing treatment effects of multiple interventions: an introduction. Rheumatol Int 2014;34:1489-96.

37 Chaimani A, Higgins JPT, Mavridis D, et al. Graphical tools for network meta-analysis in STATA. PLoS One 2013;8:e76654.

38 White IR, Barrett JK, Jackson D, et al. Consistency and inconsistency in network meta-analysis: model estimation using multivariate metaregression. Res Synth Methods 2012;3:111-25.

39 Egger M, Davey Smith G, Schneider M, et al. Bias in meta-analysis detected by a simple, graphical test. BMJ 1997;315:629-34.

40 Guyatt G, Oxman AD, Akl EA, et al. GRADE guidelines: 1. Introduction-GRADE evidence profiles and summary of findings tables. J Clin Epidemiol 2011;64:383-94.

41 Rihal CS, Naidu SS, Givertz MM, et al. 2015 SCAI/ACC/HFSA/STS clinical expert consensus statement on the use of percutaneous mechanical circulatory support devices in cardiovascular care: endorsed by the American heart Assocation, the cardiological Society of India, and Sociedad Latino americana de Cardiologia Intervencion; affirmation of value by the Canadian association of interventional Cardiology-Association Canadienne de Cardiologie d'intervention. J Am Coll Cardiol 2015;65:e7-26.

42 Martucci G, Panarello G, Occhipinti G, et al. Impact of cannula design on packed red blood cell transfusions: technical advancement to improve outcomes in extracorporeal membrane oxygenation. $J$ Thorac Dis 2018;10:5813-21. 